\title{
DETERMINATION OF ALL GENERAL HOMOGENEOUS POLYNOMIALS EXPRESSIBLE AS DETERMINANTS WHOSE ELEMENTS ARE HOMOGENEOUS POLYNOMIALS *
}

\section{H. S. EVERETT}

1. Introduction. Dickson $\dagger$ has shown that every binary and every ternary form, every quaternary quadratic, and a sufficiently general quaternary cubic form can be expressed as a determinant with linear elements, and that this property does not hold for any other form which is the general one of its degree and number of variables. The present paper is a generalization from the case of determinants with linear elements to that of determinants with elements of higher degrees.

In the first part it is proved that if the number of variables is greater than three, no general form of degree $r s$ is expressible determinantally, that is, as a determinant of order $r$ with homogeneous elements of degree $s, s \geqq 2$. The case of quadratic elements is considered in $\S 2$. The second part treats the determinantal expression of binary and ternary forms. In $\S 5$ binary forms are expressed rationally as determinants. In $\S 6$ a general procedure is set up for ternary forms and the remaining sections show that this procedure is successful for ternary quartic and sextic forms.

\section{Forms with $n>3$ ARE NOT eXPRessible determinantally}

2. Theorem 1. When the number of terms in the general form of degree $2 r$ in $n$ variables $(n \geqq 2)$ exceeds $\left[\frac{1}{2} n(n+1)-2\right] r^{2}+2$, the form is not expressible as a determinant whose elements are quadratic forms.

Let $D$ be any $r$-rowed determinant with quadratic elements homogeneous in the $n$ variables $x_{1}, \cdots, x_{n}$, which represents the general form of degree $2 r$. Its matrix $M$ may be written in the form

$$
M=x_{1}^{2} M_{11}+x_{1} x_{2} M_{12}+\cdots+x_{1} x_{n} M_{1 n}+x_{2}^{2} M_{22}+\cdots+x_{n}^{2} M_{n n},
$$

where each $M_{i j}$ is a matrix whose $r^{2}$ elements are constants. We proceed to simplify the matrix $M$ by the method of matrix multiplication employed by Dickson. $\ddagger$

* Presented to the Society, December 28, 1921.

$\dagger$ These Tran s a c ti o n s, vol. 22 (1921), p. 167, hereinafter referred to as D.

$\ddagger$ D, p. 168. 
Since $D$ represents the general form, $x_{1}^{2 r}$ is present, whence the determinant of $M_{11}$ is not zero. Thus $M_{11}$ has an inverse $M_{11}^{-1}$ such that $M_{11} M_{11}^{-1}$ is the identity matrix $I$. The new matrix

$$
N=M M_{11}^{-1}=x_{1}^{2} I+x_{1} x_{2} N_{12}+\cdots+x_{n}^{2} N_{n n} \quad\left(N_{i j}=M_{i j} M_{11}^{-1}\right)
$$

has as its determinant $D /\left|M_{11}\right|$ and has unity as the coefficient of $x_{1}^{2 r}$. Next we choose a matrix $B$ such that $B^{-1} N_{12} B$ shall have a canonical form $P_{12}$. Then our matrix reduces to

$$
P=B^{-1} N B=x_{1}^{2} I+x_{1} x_{2} P_{12}+\cdots+x_{n}^{2} P_{n n} \quad\left(P_{i j}=B^{-1} N_{i j} B\right) .
$$

In contrast with the case of linear elements, we cannot show in our case of quadratic elements that the roots $\lambda_{1}, \cdots, \lambda_{r}$ of the characteristic equation of $N_{12}$ are all distinct. Hence we permit $P_{12}$ to have the most general form* with $t$ distinct $\lambda$ 's, $t \leqq r$. Further normalization of $P$ is effected by the matrix $K$ commutative with $P_{12}$ as defined by Dickson. $\dagger$ In $K^{-1} P_{13} K$ we can specialize $r-1$ elements.

In case $t=r$ no further specialization is possible. We have then, if we count the factor removed from $D$ and the $r$ distinct $\lambda$ 's of $P_{12}$,

$$
1+r+\left[\frac{1}{2} n(n+1)-2\right] r^{2}-(r-1)
$$

or $\left[\frac{1}{2} n(n+1)-2\right] r^{2}+2$ parameters in the modified matrix of $D$. In case $t<r$ the true number of parameters is less than that given above because we then have $t<r$ distinct $\lambda$ 's, and because further normalization is possible since the matrix $K$ is not the most general matrix commutative with $P_{12}$. Therefore, the number of parameters given above is the true maximum and our theorem follows.

3. Theorem 2. When the number of terms in the general form of degree $r s$ in $n$ variables $(n \geqq 2)$ exceeds

$$
S=\left[\frac{n(n+1) \cdots(n+s-1)}{s !}-2\right] r^{2}+2,
$$

it is not expressible as a determinant whose elements are s-ic forms.

The argument in $\S 2$ applies when $D$ is a determinant of order $r$ whose elements are forms of degree $s \geqq 2$ in $n$ variables, except as to the number of terms in the matrix $M$. This number is $[n(n+1) \cdots(n+s-1)] / s !$.

4. Theorem 3. If $\ddagger n>3, s \geqq 2$, the general $n$-ary rs-ic form cannot be expressed as a determinant of order $r$ whose elements are $n$-ary s-ic forms.

The number of terms in the general form of degree $r s$ in $n$ variables is

$$
\left(\begin{array}{c}
r s+n-1 \\
r s
\end{array}\right)=\frac{(r s+1) \cdots(r s+n-1)}{(n-1) !} .
$$

* D, p. 178; Dickson, Linear Groups, 1901, p. 223; Bôcher, Higher Algebra, p. 293.

† D, p. 179.

$\ddagger$ For $s=1$ see D, Theorem II, p. 171. 
Then by $\S 3$ the general form of degree $r s$ in $n$ variables $(n \geqq 2$ ) cannot be expressed as a determinant with $s$-ic elements if

$$
\frac{(r s+1) \cdots(r s+n-1)}{(n-1) !}>S .
$$

For $n=2$ and $n=3$, respectively, (1) becomes $0>(r-1)[(s-1) r-1]$ and $0>(r-1)[(3 s-2) r-2]$, which hold for no value of $r$ when $s \geqq 2$.

Now (1) holds if

$$
\frac{(r s+1)(r s+2)(2 s+3) \cdots(2 s+n-1)}{(n-1) !}>S
$$

holds, where the left member of (2) has been obtained from that of (1) by replacing $r$ by 2 everywhere except in the first two factors. We consider this relation first for $s=2$. If $n \geqq 7, r \geqq 2$, we can cancel from the numerator and denominator of the left member the factors $7, \cdots,(n-1)$ (which are absent if $n=7$ ), and then (2) holds if

$$
\begin{aligned}
& \frac{(2 r+1)(2 r+2) n(n+1)(n+2)(n+3)}{6 !} \\
& \geqq \frac{1}{4}\left[\frac{n(n+1)}{2}-2\right](2 r+1)(2 r+2),
\end{aligned}
$$

since the right member exceeds the value $\left[\frac{1}{2} n(n+1)-2\right] r^{2}+2$ of $S$ for $s=2$. The last inequality holds if

$$
n(n+1)(n+2)(n+3) / 180 \geqq \frac{1}{2} n(n+1)-2 ;
$$

that is, if $n(n-7)(n+1)(n+12)+360 \geqq 0$, which is obviously true for $n \geqq 7$. For $n=4, n=5, n=6$, respectively, (2) becomes

$$
\begin{gathered}
(2 r-1)(2 r-2)(2 r-3)>0, \quad(r-1)\left(4 r^{3}+24 r^{2}-19 r+6\right)>0, \\
(r-1)\left(8 r^{4}+68 r^{3}+238 r^{2}-107 r+30\right)>0,
\end{gathered}
$$

each of which evidently holds for $r \geqq 2$. So (2) holds for $s=2, r \geqq 2, n \geqq 4$.

We proceed now to show by induction on $s$ that (2) and hence (1) holds for $s \geqq 2, r \geqq 2, n \geqq 4$. We assume that (2) holds for $r \geqq 2, n \geqq 4$, and any given value of $s$. We wish to show that (2) implies the corresponding inequality with $s$ replaced by $s+1$, viz.,

$$
\begin{aligned}
& \frac{[(s+1) r+1][(s+1) r+2](2 s+5) \cdots(2 s+n+1)}{(n-1) !} \\
& \quad>\left[\frac{n(n+1) \cdots(n+s)}{(s+1) !}-2\right] r^{2}+2 .
\end{aligned}
$$


To derive (3) from (2) we must add to the right member of (2)

$$
\begin{array}{r}
{\left[\frac{n(n+1) \cdots(n+s)}{(s+1) !}-\frac{n(n+1) \cdots(n+s-1)}{s !}\right] r^{2}} \\
=\frac{(n-1) n(n+1) \cdots(n+s-1)}{(s+1) !} r^{2},
\end{array}
$$

and to the left member

where

$$
\frac{(2 s+5) \cdots(2 s+n-1)}{(n-1) !}\left(a r^{2}+b r+c\right),
$$

$$
\begin{aligned}
& a=4(n-1) s^{3}+\left(n^{2}+9 n-4\right) s^{2}+2\left(n^{2}+3 n+1\right) s+n(n+1), \\
& b=12(n-2) s^{2}+3\left(n^{2}+5 n-10\right) s+3 n(n+1), \\
& c=8(n-3) s+2\left(n^{2}+n-12\right) .
\end{aligned}
$$

To establish the induction we need

$$
\begin{aligned}
(2 s+5) \cdots(2 s+n-1)\left(a r^{2}+b r+c\right) /(n-1) & \\
& \geqq(s+2) \cdots(s+n-1) r^{2} .
\end{aligned}
$$

Since the $s$-factors on the left are each greater than the corresponding $s$-factors on the right, and since $b r+c>0$ for $n \geqq 3$, (4) is implied by

$$
a /(n-1) \geqq(s+2)(s+3)(s+4) .
$$

Now (5) holds for $n \geqq 4$ if it holds for $n=4$ since the coefficients of $s$ are all positive for these values of $n$. But for $n=4$, (5) becomes

$$
9 s^{3}+21 s^{2}-20 s-52 \geqq 0,
$$

which obviously holds for $s \geqq 2$. Our induction is therefore complete and Theorem 3 follows.

\section{BINARY AND TERNARY FORMS EXPRESSED DETERMINANTALLY}

5. Binary forms. Since the binary form of degree $r s$ can be factored into $r s$ linear factors, it is expressible as an $r$-rowed determinant each of whose diagonal elements is a product of $s$ linear factors, and whose elements outside the main diagonal are zero. Dickson has expressed the binary form rationally as a determinant with linear elements.* Every binary form of degree $r s$ can be expressed rationally as a determinant of order $r$ with $s$-ic elements:

${ }^{*} \mathrm{D}, \mathrm{p}$. 175. This dęterminant can be reduced by elementary transformations to the present one. 


$$
\begin{aligned}
& a_{0} x^{r s}+a_{1} x^{r s-1} y+\cdots+a_{r s-1} x y^{r s-1}+a_{r s} y^{r s}=
\end{aligned}
$$

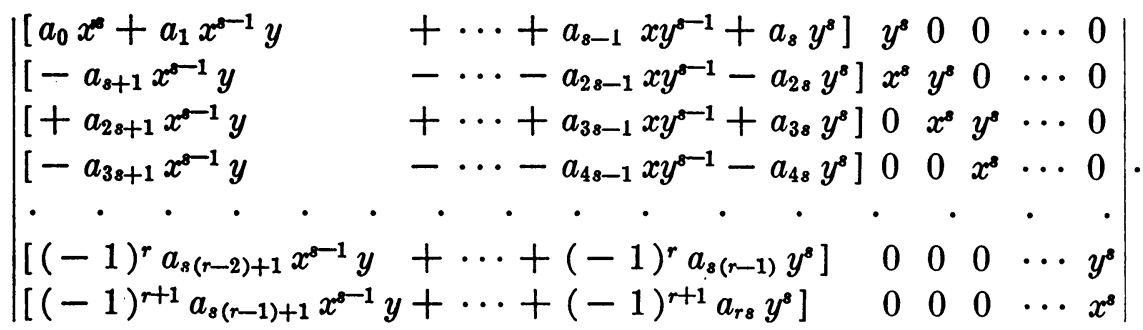

6. Ternary quartic form. The general ternary quartic may be given the form

$$
a^{2} b^{2}+c^{2} d^{2}+e^{2} f^{2}-2 a b c d-2 a b e f-2 c d e f,
$$

where $a$ and $b, c$ and $d, e$ and $f$, are three pairs of bitangents of a Steiner set.* This function equals the determinant

$$
\left|\begin{array}{cc}
(a b-c d-e f) & 2 c d \\
2 e f & (a b-c d-e f)
\end{array}\right| .
$$

The determination of the bitangents depends, however, upon the solution of equations of high order. In the case of the general ternary quartic form with no repeated factor Dickson's determinant $\dagger$ of order $r=4$ with linear elements can be reduced to a determinant of order $r=2$ with elements of degree $s=2$ by the following elementary transformations. Multiply the third row and divide the fourth column by $X_{4}+c_{44} z$; multiply the second column and divide the first row by $X_{1}+c_{11} z$; multiply the first column by $-z$ and add to the second column; multiply the fourth row by $-z$ and add to the third row; multiply the second row and divide the second column by $z$. The resulting determinant is

$$
\left|\begin{array}{cc}
l_{1} l_{2}-c_{21} z^{2} & z^{2} \\
l_{1}\left(c_{32} l_{4}-c_{42} z\right)-z\left(c_{31} l_{4}-c_{41} z\right) & l_{3} l_{4}-c_{43} z^{2}
\end{array}\right|,
$$

where $l_{i}=X_{i}+c_{i i} z$. No elementary transformations have been found which accomplish a similar reduction of Dickson's determinant of order $r s>4$.

7. General ternary form. The remainder of this paper is concerned with a method of expressing a sufficiently general ternary form $f$ of degree $r s$ as a determinant of order $r$ whose elements are $s$-ic forms. We may assume that $f$ is irreducible. For, if $f=f_{1} f_{2}$ where $f_{1}$ and $f_{2}$ are of degree $r_{1} s$ and $r_{2} s$ respectively and expressible as determinants of order $r_{1}$ and $r_{2}$ whose matrices are $M_{1}$ and $M_{2}$, then $f$ equals the determinant of the matrix

$$
\left(\begin{array}{ll}
M_{1} & O \\
O & M_{2}
\end{array}\right)
$$

* Miller, Blichfeldt, and Dickson, Finite Groups, 1916, p. 355.

$\dagger \mathrm{D}, \mathrm{p} .174$. 
where $O$ is a matrix all of whose elements are zero. With Dickson* we choose a triangle of reference such that, for $z=0, f(x, y, 0)=X_{1} X_{2} \cdots X_{r s}$, where $X_{i}=x+\lambda_{i} y$ and $\lambda_{1}, \cdots, \lambda_{r s}$ are all distinct. Then

$$
f=X_{1} X_{2} \cdots X_{r s}+\sum_{k=1}^{r s} z^{k} F_{k}(y, x),
$$

where $F_{k}$ is a binary form of degree $r s-k$. We shall attempt to express $f$ as a determinant of the following special type:

$D=\left|\begin{array}{cccc}X_{1} \cdots X_{s}+z \phi_{11} & z \phi_{12} & \cdots & z \phi_{1 r} \\ z \phi_{21} & X_{s+1} \cdots X_{2 s}+z \phi_{22} & \cdots & z \phi_{2 r} \\ \cdots & \cdots & \cdots & \cdots \\ z \phi_{r 1} & z \phi_{r 2} & \cdots & X_{r s-s+1} \cdots X_{r s}+z \phi_{r r}\end{array}\right|$,

where each $\phi_{i j}$ is a ternary form of degree $s-1$. There are $\frac{1}{2}(r s+1)(r s+2)$ coefficients in $f$. In identifying $f(x, y, 0)$ with $X_{1}, \cdots, X_{r s}, r s+1$ of these coefficients have been fixed. So the identification of $D$ with $f$ involves $\frac{1}{2} r s(r s+1)$ conditions on as many coefficients of the $\phi_{i j}$. We therefore assign simple values to the remaining $\frac{1}{2} r s(r-1)$ coefficients of the $\phi_{i j}$; in each $\phi_{i j}(j>i)$ we set the first $s$ coefficients equal to zero with the exception of the first coefficient of each $\phi_{i j}(j=i+1)$ which we set equal to one.

We then expand $D$ axially and consider the identification of the terms of this expansion, arranged in ascending powers of $z$, with the corresponding terms of any given form $f$. First, the terms linear in $z$ will be identical with $z F_{1}(y, x)$ of $f$, where $F_{1}$ is any given binary form of order $r s-1$, if they are equal for the $r s$ values $x=-\lambda_{i} y(i=1, \cdots, r s)$. The resulting $r s$ conditions involve the coefficients of the $\phi_{i j}$ of $D$. As a matter of fact, it will be seen in the following sections that they uniquely determine $r s$ coefficients of the $\phi_{i i}$. Next, the terms quadratic in $z$ will be identical with $z^{2} F_{2}(y, x)$ of $f$, where $F_{2}$ is of order $r s-2$, if they are equal for $\dagger x=-\lambda_{i} y \quad(i=1$, $\cdots, r s-1)$. The resulting $r s-1$ equations involve coefficients of $D$. Proceeding in this manner to identify terms in $z^{k}(k=1, \cdots, r s)$ we obtain a system of $r s+(r s-1)+\cdots+2+1=\frac{1}{2} r s(r s+1)$ equations in as many unknown coefficients of $D$. Since these equations involve arbitrary parameters $F_{1}, \cdots, F_{r s}$, and since by the implicit function theorem $\ddagger$ solutions exist in the neighborhood of a point for which the jacobian of the equations does not vanish, the method outlined above effects the identification of $D$ with $f$ if the jacobian of these equations is not identically zero.

*D, p. 172.

$\dagger$ Or for any other choice of $r s-1$ of the $\lambda$ 's.

$\ddagger$ Bliss, Princeton Colloquium Lectures, 1913, p. 7. Attention is called in this connection to MacMillan's method of solution involving the introduction of a parameter and the expression of all solutions in power series of this parameter. See MacMillan, $\mathrm{Math}$ e $\mathrm{mat}$ is $\mathrm{che}$ Annale n, vol. 72, p. 180. 
8. Ternary quartic form. A sufficiently general irreducible ternary quartic

$$
f=X_{1} X_{2} X_{3} X_{4}+\sum_{i=1}^{4} z^{i} F_{i}(y, x), \quad X_{i}=x+\lambda_{i} y,
$$

with distinct $\lambda$ 's can be expressed as the determinant

$$
D=\left|\begin{array}{cc}
X_{1} X_{2}+\left(h_{11} x+k_{11} y+l_{11} z\right) z & \left(x+l_{12} z\right) z \\
\left(h_{21} x+k_{21} y+l_{21} z\right) z & X_{3} X_{4}+\left(h_{22} x+k_{22} y+l_{22} z\right) z
\end{array}\right| \text {. }
$$

The ten equations which arise in the identification of $D$ with $f$ follow. For brevity we write the frequently recurring expression $\lambda_{u}-\lambda_{v}$ as $(u v)$, and $k_{u v}-\lambda_{v} h_{u v}$ as $(u v w)$.

$$
\begin{aligned}
& \begin{cases}(3 i)(4 i)(11 i)=F_{1}\left(1,-\lambda_{i}\right) & (i=1,2), \\
(1 j)(2 j)(22 j)=F_{1}\left(1,-\lambda_{j}\right) & (j=3,4) ;\end{cases} \\
& \begin{cases}(3 i)(4 i) l_{11}+(11 i)(22 i)+\lambda_{i}(21 i)=F_{2}\left(1,-\lambda_{i}\right) & (i=1,2), \\
(13)(23) l_{22}+(113)(223)+\lambda_{3}(213)=F_{2}\left(1,-\lambda_{3}\right) ;\end{cases} \\
& \left(\begin{array}{ll}
(11 i) l_{22}+(22 i) l_{11}-(21 i) l_{12}+\lambda_{i} l_{21}=F_{3}\left(1,-\lambda_{i}\right) & (i=1,2) ;
\end{array}\right. \\
& l_{11} l_{22}-l_{12} l_{21}=F_{4} .
\end{aligned}
$$

The first four equations are linear and determine uniquely $k_{11}, h_{11}, k_{22}, h_{22}$, since their determinant is

where

$$
M_{4}=m_{1} m_{2}=(31)^{2}(41)^{2}(32)^{2}(42)^{2}(21)(43),
$$

$$
m_{1}=(31)(41)(32)(42)\left|\begin{array}{ll}
1 & -\lambda_{1} \\
1 & -\lambda_{2}
\end{array}\right|, \quad m_{2}=(13)(23)(14)(24)\left|\begin{array}{ll}
1 & -\lambda_{3} \\
1 & -\lambda_{4}
\end{array}\right| \text {, }
$$

and is different from zero in view of the fact that the $\lambda$ 's are distinct. Since this system falls naturally into groups of $4,3,2$, and 1 equations each, the Laplacian expansion of the jacobian of the system by minors of the last row, of the eighth and ninth rows, of the fifth, sixth, and seventh rows, and of the first four rows yields most readily the complete coefficient of the various terms. If the jacobian has its columns in the order $k_{11}, h_{11}, k_{22}, h_{22}, k_{21}, h_{21}, l_{22}, l_{11}$, $l_{21}, l_{12}$, we find on the main diagonal the only term of the expansion actually involving $l_{21} k_{22}$. Its coefficient is

where

$$
M_{4} M_{3} M_{2} M_{1}=(21)^{3}(31)^{3}(32)^{3}(41)^{2}(42)^{2}(43) \lambda_{1} \lambda_{2},
$$

$$
M_{1}=-1, \quad M_{2}=\left|\begin{array}{cc}
1 & \lambda_{1} \\
1 & \lambda_{2}
\end{array}\right|, \quad M_{3}=\left|\begin{array}{ccc}
\lambda_{1} & -\lambda_{1}^{2} & 0 \\
\lambda_{2} & -\lambda_{2}^{2} & 0 \\
\lambda_{3} & -\lambda_{3}^{2} & (13)(23)
\end{array}\right| .
$$

We may assume that $\lambda_{1} \lambda_{2} \neq 0$, since if one of the four distinct $\lambda$ 's is zero it may be taken initially to be $\lambda_{4}$. Then since the $\lambda$ 's are distinct, the coefficient of $l_{21} k_{22}$ is different from zero and the jacobian does not vanish identically.

9. Ternary sextic form, $s=3$. A sufficiently general irreducible ternary 
sextic

$$
f=X_{1} X_{2} \cdots X_{6}+\sum_{k=1}^{6} z^{k} F_{k}(y, x), \quad X_{i}=x+\lambda_{i} y,
$$

with $\lambda$ 's distinct can be expressed as the determinant $D$ with cubic elements

$$
D=\left|\begin{array}{cc}
X_{1} X_{2} X_{3}+z \phi_{11} & z \phi_{12} \\
z \phi_{21} & X_{4} X_{5} X_{6}+z \phi_{22}
\end{array}\right|,
$$

where $\phi_{i j}=a_{i j} x^{2}+b_{i j} x y+c_{i j} y^{2}+d_{i j} x z+e_{i j} y z+f_{i j} z^{2}$, and where in particular $a_{12}=1, b_{12}=c_{12}=0$. The identification of $D$ with $f$ involves the following 21 equations. We use the abbreviated notations $(u v)=\lambda_{u}-\lambda_{v}$, $(u v w)=e_{u v}-\lambda_{w} d_{u v}$, and $[u v w]=a_{u v} \lambda_{w}^{2}-b_{u v} \lambda_{w}+c_{u v}$.

$$
\begin{aligned}
& \begin{cases}(4 i)(5 i)(6 i)[11 i]=F_{1}\left(1,-\lambda_{i}\right) & (i=1,2,3),\end{cases} \\
& \left\{(1 j)(2 j)(3 j)[22 j]=F_{1}\left(1,-\lambda_{j}\right) \quad(j=4,5,6) ;\right. \\
& \left\{(4 i)(5 i)(6 i)(11 i)+[11 i][22 i]-\lambda_{i}^{2}[21 i]=F_{2}\left(1,-\lambda_{i}\right)(i=1,2,3)\right. \text {, } \\
& \left\{(1 j)(2 j)(3 j)(22 j)+[11 j][22 j]-\lambda_{j}^{2}[21 j]=F_{2}\left(1,-\lambda_{j}\right) \quad(j=4,5)\right. \text {; } \\
& \left\{\begin{array}{c}
(4 i)(5 i)(6 i) f_{11}+[11 i](22 i)+[22 i](11 i) \\
-\lambda_{i}^{2}(21 i)-[21 i](12 i)=F_{3}\left(1,-\lambda_{i}\right) \quad(i=1,2,3), \\
(14)(24)(34) f_{22}+[114](224)+[224](114) \\
-\lambda_{4}^{2}(214)-[214](124)=F_{3}\left(1,-\lambda_{4}\right) ;
\end{array}\right. \\
& {[11 i] f_{22}+[22 i] f_{11}+(11 i)(22 i)-\lambda_{i}^{2} f_{21}-[21 i] f_{12}}
\end{aligned}
$$$$
-(12 i)(21 i)=F_{4}\left(1,-\lambda_{i}\right) \quad(i=1,2,3) ;
$$$$
(11 i) f_{22}+(22 i) f_{11}-(12 i) f_{21}-(21 i) f_{12}=F_{5}\left(1,-\lambda_{i}\right) \quad(i=1,2) \text {; }
$$
$f_{11} f_{22}-f_{12} f_{21}=F_{6}$.

With the columns of the jacobian arranged in the order $a_{11}, b_{11}, c_{11}, a_{22}, b_{22}$, $c_{22}, a_{21}, b_{21}, c_{21}, d_{22}, e_{22}, d_{21}, e_{21}, d_{11}, e_{11}, f_{11}, f_{22}, f_{21}, d_{12}, e_{12}, f_{12}$, we find on the main diagonal the complete term of the expansion involving $f_{21}^{3} b_{11} c_{22}^{3}$. Its coefficient is

$M_{6} M_{5} M_{4} M_{3} M_{2} M_{1}=\lambda_{1}^{2} \lambda_{2}^{2} \lambda_{3}^{2}(12)^{5}(13)^{4}(14)^{4}(15)^{3}(16)^{2}(23)^{4}$

where

$$
\times(24)^{4}(25)^{3}(26)^{2}(34)^{4}(35)^{3}(36)^{2}(45)^{2}(46)(65) \text {, }
$$

$$
\begin{gathered}
M_{1}=-1, \quad M_{2}=\left|\begin{array}{cc}
\lambda_{1} & -1 \\
\lambda_{2} & -1
\end{array}\right|, \quad M_{3}=\left|\begin{array}{lll}
1 & -\lambda_{1} & -\lambda_{1}^{2} \\
1 & -\lambda_{2} & -\lambda_{2}^{2} \\
1 & -\lambda_{3} & -\lambda_{3}^{2}
\end{array}\right|, \\
M_{4}=\left|\begin{array}{cccc}
\lambda_{1}^{3} & -\lambda_{1}^{2} & -\lambda_{1} & 1 \\
\lambda_{2}^{3} & -\lambda_{2}^{2} & -\lambda_{2} & 1 \\
\lambda_{3}^{3} & -\lambda_{3}^{2} & -\lambda_{3} & 1 \\
\lambda_{4}^{3} & -\lambda_{4}^{2} & -\lambda_{4} & 1
\end{array}\right|, \quad M_{5}=\left|\begin{array}{lll}
-\lambda_{1}^{4} & \lambda_{1}^{3}-\lambda_{1}^{2} \\
-\lambda_{2}^{4} & \lambda_{2}^{3} & -\lambda_{2}^{2} \\
-\lambda_{3}^{4} & \lambda_{3}^{3} & -\lambda_{3}^{2}
\end{array}\right| \cdot\left|\begin{array}{ll}
-\lambda_{4} & 1 \\
-\lambda_{5} & 1
\end{array}\right|, \\
M_{6}=\left|\begin{array}{ccc}
\lambda_{1}^{2} & -\lambda_{1} & 1 \\
\lambda_{2}^{2} & -\lambda_{2} & 1 \\
\lambda_{3}^{2} & -\lambda_{3} & 1
\end{array}\right| \cdot\left|\begin{array}{lll}
\lambda_{4}^{2} & -\lambda_{4} & 1 \\
\lambda_{5}^{2} & -\lambda_{5} & 1 \\
\lambda_{6}^{2} & -\lambda_{6} & 1
\end{array}\right| .
\end{gathered}
$$


We may assume that $\lambda_{1} \lambda_{2} \lambda_{3} \neq 0$, since if one of the six distinct $\lambda$ 's is zero, it may be taken initially to be $\lambda_{6}$. Then since the $\lambda$ 's are distinct this coefficient is different from zero and the jacobian does not vanish identically.

10. Ternary sextic form, $s=2$. A sufficiently general ternary sextic (6) can also be expressed as the determinant $D$ with quadratic elements

$$
D=\left|\begin{array}{ccc}
X_{1} X_{2}+z \phi_{11} & z \phi_{12} & z \phi_{13} \\
z \phi_{21} & X_{3} X_{4}+z \phi_{22} & z \phi_{23} \\
z \phi_{31} & z \phi_{32} & X_{5} X_{6}+z \phi_{33}
\end{array}\right|,
$$

where $\phi_{i j}=h_{i j} x+k_{i j} y+l_{i j} z$ and $h_{12}=h_{23}=1, h_{13}=k_{12}=k_{13}=k_{23}=0$. Let $\Delta$ be the determinant $\left|l_{i j}\right|$ of third order, and let $L_{i j}$ be the minor of $l_{i j}$ in this determinant. In the following 21 equations which arise in the identification* of $D$ with (6) we use the abbreviations

$$
(u v)=\lambda_{u}-\lambda_{v}, \quad(u v w)=k_{u v}-\lambda_{w} h_{u v}:
$$

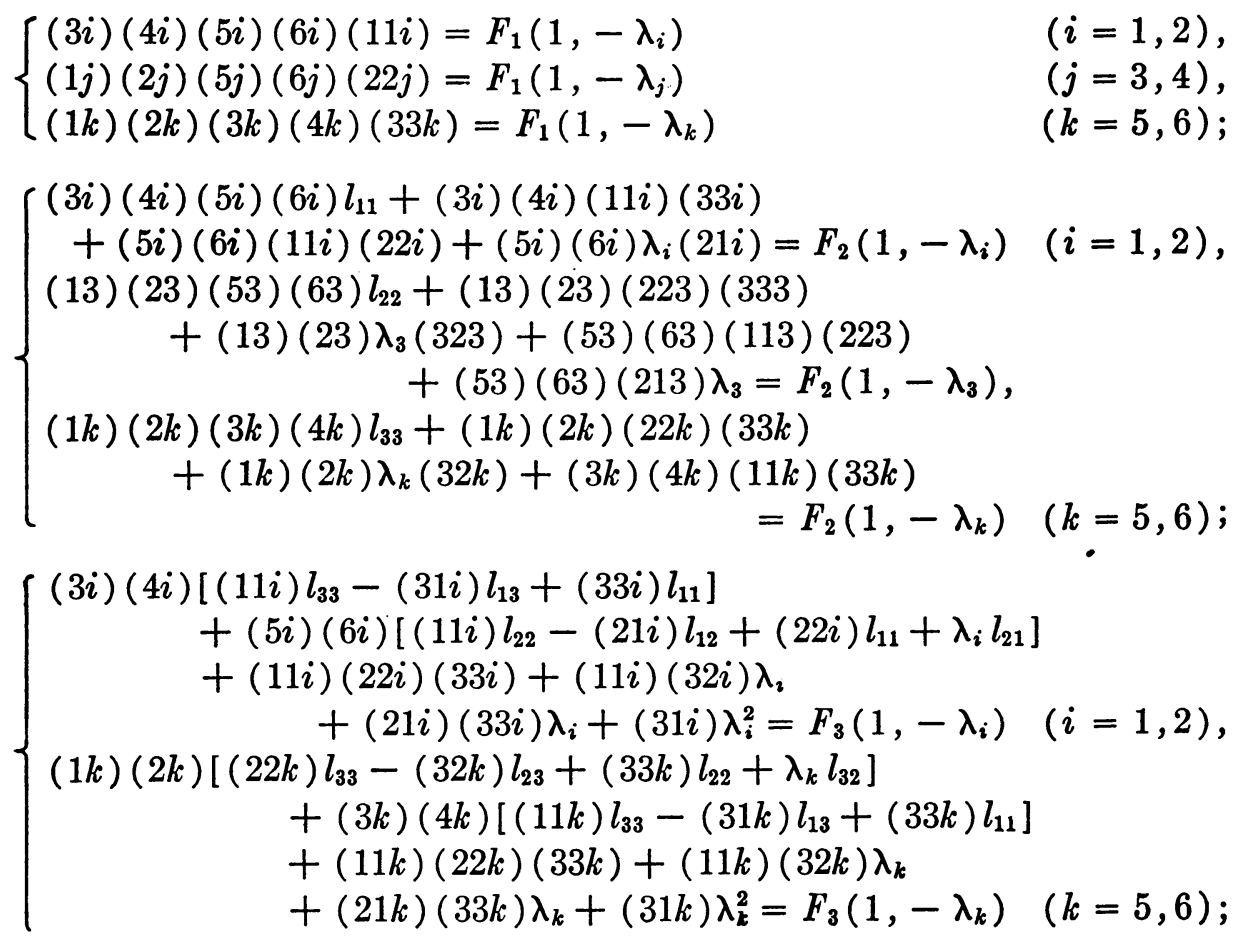

* See second footnote, $\$ 7$. Here it was found advantageous to select the $\lambda$ 's in the order $\lambda_{1}, \lambda_{2}, \lambda_{6}, \lambda_{6}, \lambda_{8}, \lambda_{8}$, since this selection gives the minimum number of terms in certain equations. Thus, the tenth and eleventh equations contain fewer terms than does the ninth equation. 


$$
\left\{\begin{array}{rl}
(5 i)(6 i) L_{33} & +(3 i)(4 i) L_{22}+l_{13}[(21 i)(32 i)-(22 i)(31 i)] \\
& -l_{23}\left[(11 i)(32 i)+\lambda_{i}(31 i)\right] \\
& +l_{33}\left[(11 i)(22 i)+\lambda_{i}(21 i)\right] \\
& +\lambda_{i}\left[(11 i) l_{32}-(31 i) l_{12}+(32 i) l_{11}+\lambda_{i} l_{31}\right] \\
& +(33 i)\left[(11 i) l_{22}-(21 i) l_{12}+(22 i) l_{11}+\lambda_{i} l_{21}\right] \\
& =F_{4}\left(1,-\lambda_{i}\right)
\end{array} \quad(i=1,2) ;\right.
$$

Similarly for $i=6$ with first term replaced by (16) (26) $L_{11}$;

$$
\begin{aligned}
& \text { (11i) } L_{11}-(21 i) L_{21}+(31 i) L_{31}+\lambda_{i} L_{12}+(22 i) L_{22} \\
& -(32 i) L_{32}+\lambda_{i} L_{23}+(33 i) L_{33}=F_{5}\left(1,-\lambda_{i}\right) \quad(i=1,2) \text {; } \\
& \Delta=F_{6} \text {. }
\end{aligned}
$$

With the columns of the jacobian arranged in the order $k_{11}, h_{11}, k_{22}, h_{22}$, $k_{33}, h_{33}, k_{21}, h_{21}, l_{22}, k_{32}, h_{32}, k_{31}, h_{31}, l_{33}, l_{32}, l_{11}, l_{21}, l_{31}, l_{12}, l_{23}, l_{13}$, we find on the main diagonal the complete term of the expansion involving $l_{21}^{2} l_{32}^{2} h_{11} k_{22}^{2} k_{33}^{3}$. Its coefficient is

$$
\begin{aligned}
M_{6} M_{5} M_{4} M_{3} M_{2} M_{1}=\lambda_{1}^{3} \lambda_{2}^{3} \lambda_{5} \lambda_{6}(12)^{5}(13)^{3}(14)^{2}(15)^{5}(16)^{6} \\
\\
\quad \times(23)^{3}(24)^{2}(25)^{5}(26)^{6}(34)(35)^{3}(36)^{3}(45)^{2}(46)^{2}(56)^{3},
\end{aligned}
$$

where

$$
\begin{aligned}
& M_{1}=1, \quad M_{2}=-\left|\begin{array}{cc}
1 & \lambda_{1} \\
1 & \lambda_{2}
\end{array}\right|, \quad M_{3}=\left|\begin{array}{ccc}
1 & \lambda_{1} & \lambda_{1}^{2} \\
1 & \lambda_{2} & \lambda_{2}^{2} \\
1 & \lambda_{6} & \lambda_{6}^{2}
\end{array}\right|, \\
& M_{4}=\left|\begin{array}{cccc}
\lambda_{1}^{2} & -\lambda_{1}^{3} & 0 & 0 \\
\lambda_{2}^{2} & -\lambda_{2}^{3} & 0 & 0 \\
\lambda_{5}^{2} & -\lambda_{5}^{3} & (15)(25) & (15)(25) \lambda_{5} \\
\lambda_{6}^{2} & -\lambda_{6}^{3} & (16)(26) & (16)(26) \lambda_{6}
\end{array}\right|, \\
& M_{5}=(13)(23)(53)(63)(51)^{2}(61)^{2}(52)^{2}(62)^{2} \lambda_{1} \lambda_{2} \lambda_{5} \lambda_{6} \\
& \times\left|\begin{array}{cc}
1 & -\lambda_{1} \\
1 & -\lambda_{2}
\end{array}\right| \cdot\left|\begin{array}{cc}
1 & -\lambda_{5} \\
1 & -\lambda_{6}
\end{array}\right| \\
& M_{6}=(31)^{2}(41)^{2}(51)^{2}(61)^{2}(32)^{2}(42)^{2}(52)^{2}(62)^{2}(53)^{2} \\
& \times(63)^{2}(54)^{2}(64)^{2}\left|\begin{array}{cc}
1 & -\lambda_{1} \\
1 & -\lambda_{2}
\end{array}\right| \cdot\left|\begin{array}{cc}
1 & -\lambda_{3} \\
1 & -\lambda_{4}
\end{array}\right| \cdot\left|\begin{array}{cc}
1 & -\lambda_{5} \\
1 & -\lambda_{6}
\end{array}\right| .
\end{aligned}
$$

We may assume that $\lambda_{1} \lambda_{2}{ }_{5} \lambda_{6} \neq 0$ since if one of the six distinct $\lambda$ 's is zero it may be taken initially to be $\lambda_{4}$. Then since the $\lambda$ 's are distinct the coefficient of this term is different from zero and the jacobian does not vanish identically.

No attempt has been made to prove that for ternary forms higher than the sextic the corresponding jacobian is not identically zero.

BUCKNELL UNIVERSITY,

Lewisburg, Pa. 